Voix et Images

voixetimages

\title{
François Hertel, curieux homme
}

\section{Gilles Thérien}

Volume 2, numéro 1, septembre 1976

Fernand Leduc

URI : https://id.erudit.org/iderudit/200020ar

DOI : https://doi.org/10.7202/200020ar

Aller au sommaire du numéro

\section{Éditeur(s)}

Les Presses de l'Université du Québec

\section{ISSN}

0318-9201 (imprimé)

1705-933X (numérique)

Découvrir la revue

\section{Citer cet article}

Thérien, G. (1976). François Hertel, curieux homme. Voix et Images, 2(1), 47-59. https://doi.org/10.7202/200020ar d'utilisation que vous pouvez consulter en ligne.

https://apropos.erudit.org/fr/usagers/politique-dutilisation/ 


\section{François Hertel, curieux homme}

Vouloir parcourir une œuvre de Hertel et en rendre compte sont deux activités difficilement conciliables. On ne sait jamais de quel côté il faut regarder: côté écriture ou côté lecture? La difficulté s'accroît quand il s'agit de vouloir faire une analyse plus rigoureuse d'une ceuvre qui n'est ni roman, ni poésie, ce qui est le cas de Mystère cosmique et condition humaine ${ }^{1}$, sorte d'essai philosophique écrit au «je». Pourtant l'occasion est excellente de se mettre à l'écoute d'une voix qui risque l'éraillement à force de crier dans le désert. De plus, l'effort méthodologique obligatoire peut être profitable face à un genre mal exploré.

Mystère cosmique et condition humaine est en date le dernier livre de François Hertel, publié aux Éditions La Presse, grâce à une subvention du Conseil des arts. Il paraît dans la collection "Écrivains des deux mondes ", ce qui peut faire rire ou pleurer. Selon. On s'imagine en regardant la maquette que les deux cent cinquante pages qui suivent sauront éclairer d'un nouveau jour ce mystère que l'on dit cosmique. Une lecture attentive permettra de se rendre mieux compte de ce qu'il en est.

\section{LE TITRE}

Qu'il soit pris en lui-même ou qu'il soit examiné en fonction de l'ensemble des titres de l'œuvre d'Hertel, il est possible d'y voir à la fois un retour vers l'origine de la carrière de l'écrivain et une reprise des préoccupations de celui-ci. Le titre a l'avantage (ou l'inconvénient) de permettre toutes les permutations:

forme actuelle A Mystère cosmique et condition humaine formes permutées $A_{1}$ Mystère humain et condition cosmique

$B_{1}$ Cosmos mystérieux et humanité conditionnée

$\mathrm{B}_{2}$ Cosmos conditionné et humanité mystérieuse.

Ces permutations mettent l'accent sur des couples d'opposition, chacun étant parfaitement logique: 
mystère cosmique / mystère humain condition cosmique / condition humaine cosmos conditionné / humanité conditionnée.

En fait, ne s'opposent que les termes "mystère" et "condition". "Cosmos" et "humain" sont à faible dénotation mais permettent dans leur rôle prédicatif une connotation maximale, lieu de toutes les idéologies. En outre, la conjonction des deux syntagmes nominaux est parfaitement englobante et on ne voit pas très bien ce qui aurait pu être éliminé, sauf Dieu et les légumes, et encore! En somme le titre veut tellement tout dire, est si permissif au plan de la permutation des notions, qu'il ne dit rien.

Toutefois une comparaison entre ce titre et d'autres de l'œuvre de Hertel permet de retrouver une constante dans les intentions de l'auteur: " inquiétude», "méditation», "journal", "sagesse», "cosmos", "mondes chimériques". Chacun de ces termes peut rendre compte du "mystère cosmique " et alors la "condition humaine" devient le lieu du discours de l'auteur. Le titre de ce dernier ouvrage pourrait être "le Monde et Moi", titre inacceptable par sa prétention mais dont le sens rejoint bien Mystère cosmique et condition humaine.

\section{QUESTION DE MÉTHODE}

Est-il possible d'utiliser ici une grille d'analyse qui soit juste par rapport au texte? Oui, à la condition de chercher à comprendre ce que dit globalement le texte et de définir des limites et des axes à l'analyse 2 .

L'essai, tel que défini par le dictionnaire, « ouvrage littéraire en prose, de facture très libre, traitant d'un sujet qu'il n'épuise pas ou réunissant des articles divers", ne semble pas suggérer une structure très rigoureuse. Ce n'est pas ainsi que nous pouvons établir une grille d'analyse. Toutefois l'examen des diverses sortes d'essais, philosophique, historique, politique, etc., permet de poser comme hypothèse que l'essai détermine un point de vue, une thèse, un développement avec preuves, un caractère factuel qui semble délimiter ce qui est de ce qui n'est pas. L'essayiste est toujours le narrateur, même s'il se cache sous des formes syntaxiques plus objectives $^{3}$. L'écart entre le narrateur et l'écrivain est aussi un écart minimal. II serait possible de concevoir le fonctionnement de l'essai comme la poursuite d'une vérité pour laquelle une rhétorique de la persuasion est mise en place. En somme l'essayiste tente de délimiter les pour et les contre, le vrai et le faux à partir, bien entendu, de sa propre perspective. II choisit les arguments, les exemples qui prouvent ses dires et il présente ceux qui tiennent le mieux en échec les pensées adverses. C'est ce fonctionnement qui servira d'hypothèse pour l'analyse de Mystère cosmique et condition humaine.

Il est important de noter que la grille à établir est un grille d'analyse de l'écriture et non une grille de lecture. Le texte est lu, il a provoqué 
ses réactions en tant que texte, il reste à connaître son fonctionnement puisque c'est cette démarche qui permet d'articuler un texte dans un univers producteur.

Ceci dit, la lecture de l'essai de Hertel permet de proposer une première grille basée sur le couple «vrai-faux". Hertel, lui-même, en voit l'importance: "vrai ou faux, c'est le premier problème qui se pose au philosophe ${ }^{4}$. Or ces deux valeurs se posent comme suit:

1.

2.

3.
$F_{1}$

$F_{1}$

$F_{2}\left(V_{1}\right)$ sont posés comme absolus.

sont mis en doute.

prennent la place de $V_{1} F_{1}$.

La première période renvoie à un Hertel croyant: “Étudiant en philosophie scolastique... je m'aperçus peu à peu qu'elle n'était pas une sagesse, au sens humaniste de ce mot, mais une préthéologie. Sans cesse, elle postule Dieu; elle a même le souci de s'aligner sur la Révélation. Toutefois, comme Dieu me paraissait un postulat évident, je m'inclinais ${ }^{5}$." La seconde période, décrite d'un même jet, laisse percer de nombreux déchirements: “... j'en viens à une révision théologique. Je m'aperçois qu'au cours des siècles... on a sollicité les textes... Je perds la foi... ${ }{ }_{\text {» }}$

La troisième période, l'actuelle, semble indiquer un changement radical dans l'itinéraire: “Une sorte de nihilisme souriant fait place à la tragique aventure. On observe une morale de modération dans les plaisirs et dans les peines, qui s'apparente d'assez près au véritable épicurisme. Voilà en gros traits, mon passage d'un certain dogmatisme à un certain ${ }^{7}$ existentialisme ${ }^{8}$." C'est l'incertitude du "certain" qui a suggéré comme hypothèse un $V_{2}\left(V_{1}\right)$, c'est-à-dire une position qui se renouvelle mais à partir de concepts qui pèsent déjà lourds dans la balance du passé. Les cheminements de Hertel ont été pénibles, l'exil inévitable. Au lieu de devoir partir vers 48 , Hertel se serait vu confier un poste prestigieux vingt ans plus tard.

C'est donc le binarisme vérité-fausseté qui nous permettra d'élaborer une grille d'analyse. Les concepts seront déterminés soit par la raison, soit par l'intuition. Ils appartiendront à la synthèse élaborée ou non. Dans le cas de l'appartenance, ils seront vrais ou faux. Ainsi Dieu, nous le verrons plus loin, demeure dans la zone d'appartenance mais comme notion fausse alors que l'épistémologie fait partie de la non-appartenance. Un tableau peut être indicatif:

\begin{tabular}{|c|c|c|c|c|c|}
\hline \multirow[t]{2}{*}{ concept/notion } & \multirow[t]{2}{*}{ Raison } & \multirow[t]{2}{*}{ Intuition } & \multicolumn{2}{|c|}{ Appartenance } & \multirow[t]{2}{*}{ Non-appartenance } \\
\hline & & & VRAI & FAUX & \\
\hline concept «a» & $x$ & & & $x$ & \\
\hline concept $" \mathrm{~b}$ " & & $\mathrm{x}$ & $x$ & & \\
\hline concept $« \mathrm{c} »$ & & & & & $x$ \\
\hline
\end{tabular}




\begin{tabular}{|c|c|c|c|c|c|}
\hline \multirow[t]{2}{*}{ concept/notion } & \multirow[t]{2}{*}{ Raison } & \multirow[t]{2}{*}{ Intuition } & \multicolumn{2}{|c|}{ Appartenance } & \multirow[t]{2}{*}{ Non-appartenance } \\
\hline & & & VRAI & FAUX & \\
\hline concept «d» & $x$ & & $x$ & & \\
\hline concept $« \theta »$ & $x$ & & & & $x$ \\
\hline concept $\propto f »$ & & $x$ & & $x$ & \\
\hline concept «g» & & $x$ & & & $x$ \\
\hline
\end{tabular}

Ce sont tous les arrangements conceptuels possibles, y compris le concept « $c$ » qui est opposé à son absence dans le texte et ne peut être obtenu que par une lecture critique. La synthèse philosophique de Hertel consisterait surtout à substituer à une première matrice intellectuelle, une seconde où nous retrouvons les mêmes facteurs mais dans un rôle inversé. Une fois l'hypothèse vérifiée, il faudra tenter de comprendre ce qui est mis en jeu par cette substitution.

Enfin, dernier élément important de l'hypothèse: le binarisme enseignant/enseigné. Hertel se place dans la situation où il doit livrer (à nouveau) un enseignement, une synthèse de sa pensée, propre, on le suppose, à rallier les esprits. Ce dialogue implicite informe tout le texte. L'utilisation du «je " et de la panoplie des possessifs contribue largement à faire apparaître ce binarisme. Nous aurons aussi à en rendre compte.

\section{LE TEXTE DANS SA TOTALITÉ}

L'examen du texte dans sa totalité pose deux problèmes, l'un à propos de son contenu, l'autre à propos de son organisation. Hertel nous prévient lui-même: "Ces études, qui étaient éparses dans plusieurs de mes ouvrages plus ou moins récents, je m'efforce de les grouper par chapitres et de les compléter et préciser par d'abondantes retouches ${ }^{9}$." II ne s'agit donc pas d'un ouvrage neuf mais bien de la collection de textes dont les uns remontent à $1947^{10}$.

Quelques textes de présentation sont nouveaux, des raccords sont mis en place entre des textes de diverses époques, des variantes corrigent l'intention des textes. Mystère cosmique et condition humaine est une mosaïque de textes qui ont déjà porté d'autres titres ou ont fait partie d'un autre type d'organisation.

Mosaïque et non collage, l'expression est importante. Nous sommes loin d'une technique d'écriture qui provoque le texte en utilisant des modes extérieurs d'intervention ${ }^{11}$. II s'agit plutôt d'une mosaïque dans laquelle l'effort d'écriture consiste principalement à faire disparaître, à gommer l'hétérogénéité des éléments épars, déjà construits. Les variantes répondent alors souvent à une nouvelle logique organisationnelle dont l'unique but est, semble-t-il, de conserver aux deux cent cinquante pages du livre la plus grande unité possible. Nous reviendrons plus loin sur les 
variantes et ce qu'elles disent (ou ne disent pas). La logique de l'ensemble est plus importante.

La division adoptée par Hertel regroupe les parties suivantes:

1. Introduction à la philosophie.

2. Suis-je existentialiste?

3. Cosmo-psychologie.

4. Esthétique et philosophie du langage.

5. Intuition ou raison raisonnante.

6. Le problème de la liberté.

7. Notes de philosophie sociale.

8. Philosophie de la vie.

9. Philosophie de l'inexplicable.

Appendice: Notes sur l'histoire des religions.

Or il suffit de reprendre cette division en l'examinant d'un peu plus près pour y découvrir en filigrane une autre division qui, selon l'appartenance ou non, règle la première division.

I. (Logique)

II. (Philosophie de la nature)

III. (Métaphysique)

IV. (Morale générale)

V. (Morale spéciale)

VI. (Théodicée)
1. Introduction à la philosophie

2. Suis-je existentialiste?

3. Cosmo-psychologie

5. Intuition ou raison raisonnante

9. Philosophie de l'inexplicable

4. Esthétique et philosophie du langage

6. Problème de la liberté

8. Philosophie de la vie

7. Notes de philosophie sociale

A. Appendice sur l'histoire des religions

L'ordre $(1,2,3,4,5,6,7,8,9, A)$ devient $(1,2,3,5,9,4,6,8,7, A)$. Or cette opération nous permet de découvrir comme base réelle de la division de l'œuvre la division traditionnelle des manuels québécois de philosophie scolastique. Seule la logique, et pour cause, est remplacée par une sorte d'introduction biographique et pédagogique genre «conseils au jeune philosophe", préoccupation qu'on retrouve aussi chez Thomas d'Aquin. Nous voici donc en face de notre première hypothèse puisque la division traditionnelle correspond au binarisme $V_{1}-F_{1}$ et il nous reste à montrer que la division actuelle est $V_{2}\left(F_{1}\right) F_{2}\left(V_{1}\right)$, c'est-à-dire fonction (modifiée) de la première.

Prenons le cas de la métaphysique. Chez Hertel, le titre est «Esthétique et philosophie du langage" mais le reste de cette partie montre bien qu'il faut faire du langage et de l'art une métaphysique. Ce qui apparaît nouveau $\left(V_{2}\right)$ est ce qui était autrefois relégué à un rôle secondaire et nous assistons à l'évacuation de «l'être en tant qu'être», ancienne vérité $\left(V_{1}\right)$ qui devient une fausseté $\left(F_{2}\right)$ : “Si toute communication vraiment enrichissante par la métaphysique traditionnelle semble interdite à l'homme 
d'aujourd'hui, n'y aurait-il pas une possibilité de communiquer par l'esthétique? C'est ici qu'intervient le rôle de la poésie 12" et: "Pour parler de l'être, il faut avoir parlé à l'être. Le langage de la poésie et de la métaphysique de demain - qui devra être poésie ou se taire - doit surgir de l'être et tenter d'atteindre l'être jusqu'à sa moelle ${ }^{13}$." II est difficile d'être plus clair: l'ancien "être en tant qu'être" demeure comme structure mais est remplacé par la poésie.

La même aventure se retrouve au niveau de la théodicée bien morte selon Hertel mais qui monopolise suffisamment d'énergie pour que la présence de son absence engendre des pages de négations ${ }^{14}$.

Devant la difficulté de trouver un principe unificateur à ces textes qui ont été, presque tous, conçus comme des fuites de l'écrasant passé, l'ancienne structure mentale est venue à point fournir les accommodements. Le projet philosophique de Hertel est une philosophie traditionnelle où les vérités et les erreurs se sont contentées de changer de parti. La logique de tout cela est difficile à percevoir. Aussi faut-il laisser parler (d'un peu plus près) les divers morceaux de la mosaïque.

\section{MOSAİQUE D'UNE VIE ET D'UNE OEUVRE}

Quiconque voudrait reconstituer l'œuvre de François Hertel, se verrait, en quelque sorte, forcé d'en appeler aux incidences biographiques. Les textes ne peuvent s'expliquer par eux-mêmes autant à cause de leur diversité qu'à cause de leur reprise à des moments différents. Ici, c'est surtout la reprise qui domine puisque les romans, poèmes, pièces de théâtre, reportages (etc.) ont été laissés de côté au profit des textes philosophiques, des essais. La reprise qui ne se fait pas sans variantes significatives doit trouver son explication dans les changements de périodes (et d'humeur).

L'œuvre actuelle de Hertel peut se diviser en six parties. Cette division nous est suggérée par l'auteur lui-même dans le passage intitulé "Ma philosophie d'autrefois ${ }^{15}$ ". C'est moins en termes de dates qu'en termes d'œuvres que la division s'opère:

1. Période dogmatique (avant 47): avec des œuvres comme le Beau Risque, Leurs inquiétudes, Pour un ordre personnaliste.

2. Période de doute (autour de 47): avec, par exemple, le Journal d'Anatole Laplante...

3. Période du Canadien errant (les années 50): une certaine vision du Québec et du Canada transmise à l'étranger: “Un Canadien errant, ô Canada, mon pays, mes amours...

4. Période de justification (même époque et le début des années 60 ): Louis Préfontaine, apostat, Méditation théologique...

5. Période des théories du langage (à partir de 64): le Métalangage, Divagations sur le langage... 
6. Période du retour (les années 70): dont Mystère cosmique et condition humaine est un bon exemple.

Or, le problème de la reprise vient de ce que cette dernière œuvre contient des textes qui datent de la deuxième période, c'est-à-dire, de l'époque du doute. Comment peut-on après tant d'années reprendre un texte, et avec quelles conséquences? A titre d'exemple, nous prendrons le "Petit Traité du vrai en soi et au dehors", publié d'abord en 1947 dans le Journal d'Anatole Laplante ${ }^{16}$ et repris dans Mystère cosmique... en 1975. Le texte entre crochets appartient à la version 1947, les ajouts sont soulignés, les variantes grammaticales ou lexicologiques de 1975 sont entre parenthèses.

Je voudrais écrire par delà les mots un essai où il y eût uniquement des choses.

Le mot est trop avili, il fut trop stipendié. [Et puis le romantisme est venu qui l'a vidé de sens. Le romantisme qui ne fut jamais tout à fait mort tant que vivait l'auteur d' "Un jardin sur l'Oronte" et que Rostand n'était pas encore rentré dans le néant. Le mot est trop souillé.] Trop de lèvres l'ont proféré à tort et à travers, à contre sens. Je veux que l'on revienne au sens. [Á moi l'être!]

Je voudrais écrire une page dure comme le roc. [Sans mastic et sans phrases.] Et je saisirais le lecteur aux cheveux et au ventre, sans passer par les yeux et les oreilles, ou si peu que pas.

Fascination du direct, de la droiture de l'inclination (inclinaison). Arboration de la rectitude plénière.

A moi l'univers total et replié sur le centre. Tout homme est à luimême centre. Et l'excentricité de l'amour n'est qu'un effort d'attirer au centre.

Nous sommes centre. En nous tous réside la quintessence du cercle: le point central. De moi au monde jaillissent des milliards de rayons des milliards d'antennes. L'homme est un animal qui a des antennes.

Chaque homme est à lui-même le centre du monde. II recrée à son intérieur le monde à son image et se pose par l'opposition indéfinie des relations. La personne est ce qui ne se donne, ni ne se prête, ni ne s'évade. Elle est ce à quoi nul ne renonce. IY renoncer serait rompre avec l'ordre essentiel, s'écarter de la fin ultime par l'abandon du but prochain.]

II faut passer par toutes les marches de l'escalier.

Et ce bourgeon sur la personne interpellant l'être, c'est (ce qu'on appelle) le vrai. S'il n'y avait point de personne, (il n'y aurait) point de vrai [autre que le Vrai en soi]. Abolissons le moi tangent au monde par l'œil intérieur, tout l'être demeure et tout le vrai croule. [Seul en lui-même, Dieu se profère au-dessus du chaos. Et nulle voix, de l'abîme, ne répond à son appel.] (II ne s'agit d'ailleurs que du vrai subjectif. Le vrai objectif n'existe pas.)

Note: Suivent deux bonnes pages de texte où il est question de Vrai en soi, de l'Étre en soi, de Dieu, etc., et qui se termine par la phrase suivante: "Le reste est opinion". 
Revenons à la personne, centre d'attribution, lieu géométrique de la pensée assimilée. C'est parce qu'il y a des personnes qu'il y a (ce qu'on nomme) des vérités. Que d'une personne un rayon parte, qu'un phare s'allume qui tente d'illuminer un coin du monde, et c'est ce qu'on appelle penseur. [Que cet homme interpelle la création, qu'il convie d'un rythme sien le concert à s'ébranler et voilà ce que l'on nomme génie.]

L'exemple choisi est particulièrement long. II permet toutefois de considérer divers problèmes impliqués par cette reprise qui fait coïncider 1947 et 1975.

Notons que le texte n'a subi que des modifications de parties. La phrase demeure fondamentalement la même, la rhétorique aussi. Les modifications les plus importantes portent sur l'existence d'un discours théocentrique en 1947, discours qui n'est plus assumé en 1975. La suppression de «seul en lui-même, Dieu se préfère au dessus du chaos. Et nulle voix, de l'abîme, ne répond à son appel” est significative. En 1975, le «nihiliste souriant" souhaite effacer la trace de Dieu. Voilà qui est bien mais il ne suffit pas, semble-t-il, de faire disparaître. II faut, tant pour l'équilibre du paragraphe que pour celui de la pensée, compenser cette perte. Le nouveau texte ne manque pas d'étrangeté: "Abolissons le moi..., tout l'être demeure et tout le vrai croule. II ne s'agit d'ailleurs que du vrai subjectif. Le vrai objectif n'existe pas. "L'ambiguïté de l'être demeure: quel est-il ? L'ambiguïté du «vrai " est réduite mais un peu à la manière d'une excuse. En fait, “le vrai " a un sens qui s'apparente à “la Vérité, à Dieu». N'est-il pas écrit dans la version 1947: «Dieu, vérité absolue. Et combien relatives les autres vérités 17 ! ”

Au premier niveau de lecture, le fond du texte demeure le même. Toutefois, l'ancienne "Vérité" devient fausseté et disparaît. La nouvelle "vérité" se présente alors comme la partie relativisée du premier credo. C'est bien le passage de $\left(V_{1}, F_{1}\right)$ à $V_{2}\left(F_{1}\right) F_{2}\left(V_{1}\right)$. C'est la trace de Dieu qui est effacée et le «moi» qui n'était qu'une modeste partie, centre de son propre amour, Jemeure dans la même situation mais posé comme le subjectivisme absolu. Le concept de Dieu, qui ne peut plus se réclamer ni de l'intuition ni de la raison passe de l'appartenance à la non-appartenance.

Ce n'est pourtant pas la seule lecture possible. Bien d'autres éléments surgissent: les contresens et les doubles sens par exemple. Le titre clame: «Petit traité du vrai en soi et au dehors". II y aurait un dehors et un dedans - ce qui n'est pas très nouveau - mais en outre, ces deux lieux s'opposeraient un peu comme les mots et les choses, ainsi que l'indique la première phrase. C'est dans cette opposition classique de la représentation et de la réalité que l'écriture va tracer un programme à peine voilé d'une stratégie fort peu orthodoxe.

Hertel écrit: “Je veux que l'on revienne au sens." Évidemment, il s'agit du sens/définition (meaning) des choses. Et pourtant le français fournit cette étonnante ambiguïté: les sens. On peut revenir au sens ou aux sens. II se peut même que les deux expressions s'équivalent dans la. 
recherche de l'au-delà des mots. Pure invention? Peut-être. Poursuivons la lecture. II s'agit maintenant d'attaquer le lecteur au ventre et aux cheveux (c.-a.-d. système pileux), le tout en profitant, si possible, de sa cécité et de sa surdité. Et par quel moyen? Par une «page dure». Pourquoi? A cause de la «fascination" qu'exerce «la droiture de l'inclination». Remarquons qu' «inclinaison " est venu corriger en 1975 l'inclination du désir, la pente glissante... Mais la correction ne fait que porter une plus grande insistance sur cette chose "dure, droite, directe, inclinée, pleine». Qu'on ajoute encore l'utilisation du mot "arboration" si proche et si contraire d'«abhorration» et le cheminement du désir au-delà des mots n'a pratiquement plus besoin d'être mis en images. II faut plus loin voir la personne, le centre lancer ades "rayons", des "jets", le penseur se dresse comme un phare et le génie interpelle «la création qu'il convie d'un rythme sien le concert à s'ébranler»: pour comprendre une des premières affirmations: «le mot est trop souillé ". L'absence de Dieu dans tout cela, tant en 47 qu'en 75, laisse place à l'hypothèse d'un cheminement souterrain qui viendra; en temps voulu, abattre les idoles aux pieds enflés. Le sujet de la personne devient le sujet de l'énonciation et il n'est plus étonnant de voir alors disparaître la condamnation du romantisme. Bien plus, Hertel écrira plus loin: «Le mot insignifiant - au sens étymologique - me gêne donc à travers les XVII. et XVIIIe siècles. II me semble qu'on ne commence à comprendre la valeur de l'appel nominal qu'au XIXe siècle ${ }^{18}$."

Dans «le Petit Traité...", c'est la fascination du corps qui prévaut. Les formules, incantatoires dans leur style ( $\grave{A}$ moi l'être!" "le sens") définissent le sujet en utilisant des métaphores vectorielles tirées de la géométrie d'Euclide. Malheureusement c'est le désir qui, déguisé en levier d'Archimède, permettra de recouvrer les sens au lieu du sens. Le traité a sa place en 76 . On peut seulement regretter que son contenu «désir» n'ait pas connu une plus explicite carrière. Le désir demeure lui dans la non-appartenance.

\section{LE RETOUR AU MOT}

Le paradoxe installé, nous ne nous étonnerons pas de voir, soudainement, réapparaître au-delà de la chose, le mot. La philosophie du langage occupe la plus grande partie du dernier ouvrage de Hertel. Cercle vicieux?

Du métalangage (1968) et Divagations sur le langage (1969) sont les deux principales sources de cette longue partie consacrée à l'esthétique et à la philosophie du langage. Et quand Hertel se met à table, il ne lésine pas sur les invitations. L'assemblée est impressionnante. Nous ne pouvons résister à l'idée de créer des couples: Sapho et Derrida, Victor Hugo et Lévi-Strauss, Rougier et Barthes, Saint-Thomas et Meschonnic, Hérédia et Foucault, Boileau et Bloomfield, Scève et Saussure et des centaines d'autres. Mais que faire en si bonne compagnie, sinon justement des divagations. 
L'objectif était pourtant de créer un méta-langage apte à remplacer la métaphysique. Mais le malentendu sur le «méta" demeure dans les textes de Hertel. Reprenant un des débats scolastiques, il veut faire de son langage, une sorte d'ontologie de la représentation, le "méta "étant alors ce qui vient “au-dessus" (dans l'échelle des valeurs aussi) au lieu d'un "méta" qui n'indiquerait qu'un ordre chronologique des connaissances, la «Métaphysique» d'Aristote vient après la Physique... et non audessus. Hertel oppose alors le langage objectif au langage symbolique:

contrairement au langage objectif, qui est figé et conventionnel, le langage symbolique est en route vers des symboles de plus en plus subtils, éthérés, susceptibles d'une plus vaste expressivité[...]. Le langage objectif couvre le champ du truisme, du généralement admis. Le langage symbolique cherche à étendre ce champ. II est à l'aurore du métalangage, que dis-je, il est déjà du métalangage ${ }^{19}$.

La hiérarchie des valeurs est reprise. Au bas, le pauvre langage objectif, en haut, le langage symbolique... toujours plus haut vers le métalangage. Les signifiants tendent vers une sorte de signifié ultime, mystérieux, ineffable, qu'on appelle Cosmos. Il s'agit là de l'une des nombreuses variantes du logocentrisme, récusé par tous les contemporains, sollicités ici bien à tort puisque le métalangage est le langage qui vient après,... sur...

En fait, toute cette partie avant d'être une poétique est une revue de la littérature classique et, sous le mot "structuralisme", il faut voir «humanisme »:

J'admets (avec les structuralistes) enfin que ce que l'on persiste à appeler humanisme est absolument dépassé.[...] Je ne sais pas quand l'homme disparaîtra; mais je voudrais le voir enfin «apparaître» tel qu'il est, beaucoup moins beau et moins grand qu'il ne s'est acharné à se croire, mais un peu moins idiot qu'il ne tient à le demeurer actuellement.

Que le structuralisme aboutisse à une belle forme de modestie, n'est-ce pas déjà une victoire remportée ${ }^{20}$ ?

Hertel n'en démord pas: la culture, c'est la Bible, les Grecs, les Latins, les Classiques, les Poètes reconnus. Il se joint à Mallarmé et à Valéry pour réclamer l'éclatement du langage. II ne le voit cependant qu'en fonction d'une sorte de sagesse supérieure de l'homme. L'esthétique a alors un côté sacré. Ne parle-t-il pas de profération, d'incantation. En somme, le fin du fin, le bout du symbolisme, c'est le Symbole avec un grand "S". Dieu s'est effacé mais ses attributs demeurent. Là encore nous retrouvons le déplacement de $V_{1} F_{1}$ en $V_{2}\left(F_{1}\right), F_{2}\left(V_{1}\right)$.

Dans ses Divagations sur le langage 21, Hertel avait inclus un chapitre intitulé "Se perdre dans le langage ou sonnets pour Aspasie" qu'il n'a pas repris dans Mystère cosmique... Culture oblige. Madame Aspasie sévissait du temps de Périclès, modèle archaïque de Madame de Récamier. C'est à elle que Hertel dédie quatre sonnets suivis de considérations diverses dont nous retiendrons celle-ci : "Pourquai te torturer, 'ô ma douce 
Aspasie? Bientôt, je ne serai plus qu'un souvenir perdu dans ta mémoire avide! Tu sauras qu'être aimée d'un poète, c'est être aimée des mots. Et ce sera notre commun châtiment. Cette autocritique me plaît,assez. "

Échec de la représentation? Oui - Cruel. Ressenti dans la chair. Aspasie est une créature du poète, son amour aussi. Ce mariage est voué à la stérilité. Nous pouvons alors nous demander sur quoi précisément ouvre ce fameux métalangage qui serait une sorte de prise sur le réel, sinon ce même espoir caché dans "le Petit Traité du vrai en soí...", espoir de rejoindre la chair. Hertel médite encore «Et le Verbe s'est fait chair». Le logocentrisme reprend ici une nette teinte érotique qui est refusée au lecteur du Mystère cosmique... Pudeur ou crainte de la perversité de l'enseignement?

\section{LA FIN DE L'EXIL ET L'ENSEIGNEMENT}

Hertel ne reviendra probablement jamais vivre en terre québécoise. Pourtant, avec Mystère cosmique et condition humaine, c'est un peu comme si son exil prenait subitement fin.

Une lecture attentive du livre découvre une grande sérénité - de façon générale - à l'égard du Québec, chez un auteur dont les propos ont passé par toute la gamme de l'exagération. Le passé québecois y est marqué avec détachement: "Je suis donc né au Canada français dans un pays où régnait jadis un certain dogmatisme philosophico-théologique." Le texte original est: "Je suis né... dans un pays où règne en souverain le dogmatisme philosophicothéologique ${ }^{22}$. " Ceci n'est qu'un des multiples exemples que contient le présent ouvrage.

Mis à part le fait qu'il sied mal de provoquer ceux auxquels on veut s'adresser, il faut peut-être chercher ailleurs les raisons de ce changement. L'anecdotisme ne peut fournir une réponse satisfaisante. Hertel, au-delà des anecdotes, demeure «possédé» par une mission, celle d'être un maître à penser, ce qu'il a été en son temps, et de façon brillante, pour toute une génération de nos grands hommes. Mais voilà, après 1950, la communication est devenue de plus en plus difficile et les années 60 ont brouillé presque définitivement les ondes. Hertel cherche quand même à transmettre un enseignement: "Celui qui a commencé de livrer un message, si modeste soit-il, doit-il s'arrêter le jour où son message risque de troubler des esprits qu'il a voulu orienter vers une certaine forme de calme...» et plus loin: «ll faut être juste, il faut être honnête. Ces impératifs catégoriques résonnent en moi. C'est peut-être le résultat d'un complexe. Je ne sais; mais il me serait pénible de ne pas écrire loyalement aujourd'hui des pages qui $m^{\prime}$ inquiètent ${ }^{23}$.

Hertel pense qu'il a induit en erreur des esprits. II souhaite corriger, se rétracter avec un peu la même gêne qu'un politicien trahit quand il admet s'être trompé. Sincérité, voilà l'impératif catégorique qui règle les écrits de Hertel avec tout ce que cela suppose de manipulation de la véri- 
té. L'auteur sincère n'est pas celui qui dit toujours la vérité mais bien plutôt celui qui court après. Sa punition, son châtiment, c'est la culpabilité. Le Maître qui ment est méprisable. Il est coupable envers tous ceux qu'il a enseignés. Mais que sait le Maître? Seule sa vie a une quelconque valeur de vérité. Hertel écrit au «je», raconte son aventure «spirituelle», prévient les uns, encourage les autres. Son dieu n'est ni Jupiter, ni Saturne mais Mercure, le maître de la duplicité moins par mensonge que parce qu'il est double et que la vérité est toujours en fonction de ce double.

Le message livré par Mystère cosmique... est aussi organisé autour de deux axes.

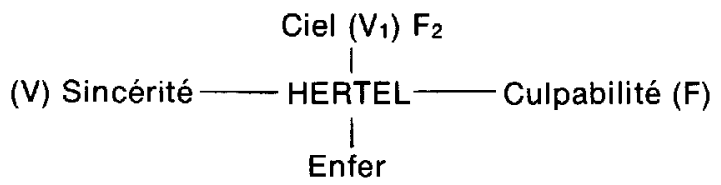

$\left(F_{1}\right) V_{2}$

Le système de déplacement des valeurs $\left(V_{1}\right) F_{2},\left(F_{1}\right) V_{2}$ tel que nous avons pu le reconstituer s'inscrit dans une sorte de déchirement entre des valeurs demeurées authentiques dans l'homme, la sincérité (V) et la culpabilité $(F)$. Seul l'enseignement peut contribuer à augmenter la sincérité au profit de la culpabilité. Le drame c'est que dans le nouvel ordre des valeurs, cela rapproche un peu plus de l'enfer et un peu moins du ciel. Les tensions sont nouées en sens inverse. Le véritable enseignement consiste alors à libérer les tensions, sachant que ceci ne peut se faire sans heurts, sans blessures. Au crépuscule de sa carrière, Hertel n'hésite pas à reprendre le fer et à le replonger dans la plaie. Mais cette plaie est surtout la sienne. Mystère cosmique et condition humaine se veut le moyen de cette ultime tentative. II ne faut pas croire que nous avons épuisé ici tous les aspects de cette mosaïque de textes. Nous avons voulu simplement tenter une explication pour cette écriture paradoxale, à l'image flamboyante, parfois cousue de fil blanc, parfois incrustée dans la chair vive.

En guise de conclusion, quoi de mieux' qu'un autre texte qui n'a pas trouvé sa place dans le dernier livre de Hertel, victime de la non-appartenance au système mais une des grandes pages qui expriment le déchirement d'une existence inquiète.

Depuis que je suis conscient, je me sais condamné à mort. Si je suis gai, c'est que j'aime crâner avec la mort, c'est aussi que je veux vivre avant de mourir. Seuls les êtres gais vivent. Les autres meurent lentement. Je suis gai dans ma vie et amer dans mes écrits. II faut bien expulser de soi cette lie du chagrin dans lequel on est né et avec lequel on mourra.

Je suis né angoissé et je mourrai dans l'angoisse: j'ai trop voulu vivre. J'ai aussi visé trop haut. Personne ne comprend mon itinéraire. Je m'y perds moi-même. Où vais-je? 
Personne ne m'a jamais connu. J'ai su me retrancher. Aux êtres que j'ai rencontrés sur mon chemin, je n'ai livré que la frange de moi-même. L'intérieur est toujours vierge.

C'est dans la nuit amère et solitaire que j'aurai le mieux appris à vivre. [...]

[...] Je suis seul en moi-même et retranché du troupeau des porcs humains. J'ai fui leur pestilentielle médiocrité. Je m'inaugure sage ${ }^{24}$.

La condition humaine, c'est le déchirement de ne pas se sentir chez soi, à l'aise, dans le mystère cosmique. L'appartenance ou la non-appartenance des concepts à un système suit leur appartenance ou non à la vie de celui qui s'est «inauguré sage». La sollicitation des choses par les mots conduit à la stérilité. Hertel promène sa lanterne et cherche un «élève »... dans un monde où il n'y a plus que des maîtres.

Gilles Thérien

1. François Hertel, Mystère cosmique et condition humaine, Montréal, La Presse, "Ecrivains des deux mondes", 1975. Ci-après, nous utiliserons $M C E C H$.

2. Les seuls essais analysés selon une méthode sémiotique se retrouvent dans Barthes, Sade, Fournier, Loyola. François Rastier pose certaines questions fort pertinentes dans Essais de sémiotique discursive et Régine Robin fait de même à propos de l'histoire dans Histoire et linguistique. Dans l'ensemble, il n'existe pas de méthode privilégiée, ce qui est au fond excellent.

3. Le parti pris de la troisième personne, par exemple.

4. $M C E C H$, p. 36.

5. Ibid., p. 26.

6. Ibid., p. 27.

7. C'est nous qui soulignons.

8. $M C E C H$, p. 29.

9. Ibid., p. 9.

10. Par exemple au Journal d'Anatole Laplante.

11. Nous pensons ici à la théorie du collage telle que définie par Aragon. Le "collé" qui vient de l'extérieur, qui doit son existence à un tout autre système, est à l'origine d'une nouvelle organisation.

12. $M C E C H$, p. 65 .

13. Ibid., p. 125.

14. Il s'agit des pages qui concernent l'histoire de la religion, la vie de Jésus (etc.) regroupées en appendice.

15. $M C E C H$, p. 13 et suiv.

16. Journal d'Anatole Laplante, Montréal, Serge Brousseau, 1947, p. 107-110. (JAP)

17. JAP, p. 109

18. $M C E C H$, p. 79

19. Ibid., p. 66.

20. Ibid., p. 113.

21. Divagations sur le langage, Paris, Éditions de la Diaspora française, 1969, p. 24.

22. Journal philosophique et littéraire, Paris, Éditions de la Diaspora française, "Les Essais", 1961, p. 9. (JPEL)

23. $M C E C H$ p. 23-24.

24. JPEL, p. 23. 